\title{
UM INDICADOR COMPARATIVO DE POBREZA MULTIDIMENSIONAL A PARTIR DOS OBJETIVOS DO DESENVOLVIMENTO DO MILÊNIO
}

\author{
Marcelo Bentes Diniz \\ Marcos Monteiro Diniz ${ }^{\dagger}$
}

\begin{abstract}
Resumo
A partir do aporte teórico da Abordagem das Capacitações e tendo como referência metodológica a técnica dos Conjuntos Fuzzy, este artigo apresenta um indicador-síntese de pobreza multidimensional para os estados brasileiros. Todavia, diferentemente de outros estudos, a contribuição deste artigo é diminuir o grau de arbitrariedade na escolha das dimensões da pobreza, considerando o cumprimento das metas dos Objetivos do Desenvolvimento do Milênio (PNUD, 2003). Os resultados apontam uma delimitação espacial bem definida no país, com os estados das Norte e Nordeste situando-se entre os dez de maiores índices, com exceção do estado do Rio Grande do Sul.
\end{abstract}

Palavras-chave: pobreza multidimensional; Objetivos do Desenvolvimento do Milênio.

\begin{abstract}
From the theoretical of Capability Approach and having as methodological reference the Fuzzy Sets technique this article presents an indexsynthesis of multidimensional poverty for the Brazilian states. However, differently from other studies, the contribution of this article is to diminish the degree of arbitrariness in choosing levels poverty dimensions, being considered the attainment of the Millennium Development Goals (PNUD, 2003). The results point to a well definite space delimitation in the country, with the states of the regions North and Northeast placing themselves among the ten highest indices, with the exception of the CityRio Grande do PlaceNameplaceSul PlaceTypeState.
\end{abstract}

Keywords: multidimensional poverty; Millennium Development Goals.

JEL classification: I32; R10.

\footnotetext{
* Professor do Programa de Pós-Graduação em Economia - UFPA. Endereçco: Trav. Rui Barbosa 770, apto 601. Reduto, Belém - PA. CEP: 66053-260. E-mail: mbdiniz2007@hotmail.com.

† Professor do Programa de Pós-Graduação em Economia - UFPA. E-mail: mdiniz@ufpa.br.
} 


\section{Introdução}

Não é plenamente consensual na literatura econômica a definição de pobreza. A conotação mais usual a "entende" como um estado de carência, a partir de um critério definido ex-ante, em geral, de caráter objetivo. Segundo Hagenaars e de Vos (1988), em seu estudo voltado a apresentar as diferentes definições existentes, é possível considerar três grandes grupos de definições: A. Pobreza é ter menos do que é objetivamente definido, mínimo absoluto; B. Pobreza é ter menos do que os outros na sociedade; e, C. Pobreza é o sentimento de não se ter o bastante para sobreviver. Assim, no primeiro caso a pobreza assume um sentido absoluto; no segundo caso, um caráter relativo e no terceiro caso, ambos.

Vale dizer que a condição relativa da pobreza permite uma interpretação tanto objetiva, por um critério previamente definido, como subjetiva, porque depende, também, de como os indivíduos se vêem dentro do contexto social. Assim, segundo Romão (1993), isto pode levar, inclusive, à possibilidade de divergência entre os dois critérios, quando se toma o indivíduo como unidade de observação a ser avaliada.

A existência de várias conotações sobre a definição da pobreza tem se traduzido em diferentes tentativas de se criar medidas-sínteses que possam expressá-las, determinando quais aspectos devem ser priorizados quanto ao nível de bem-estar dos indivíduos. Nesta direção, a abordagem das capacitações deu origem à interpretação da pobreza como formada por diferentes dimensões, englobando os elementos relativos ao chamado enfoque das necessidades básicas, mas também enfatizando a capacidade dos indivíduos, em que pese as suas escolhas quanto aos critérios de bem-estar e as formas de alcançá-los.

Assim, o que a abordagem das capacitações introduz de diferente quanto às outras abordagens é o entendimento acerca do que vêm a ser a qualidade de vida, ou melhor, qual a qualidade de vida que os indivíduos avaliam para a sua vida. Assim, a qualidade de vida não se limitaria à dotação de bens que o indivíduo possui, mas de outras condições que cercam a vida humana em sua plenitude.

Por essa via, usando a terminologia desenvolvida especialmente por Sen $(1980,1985,1999,2000)$, Sen e Foster (2001), dentro desta abordagem, a definição de um índice de pobreza multidimensional envolve um processo de avaliar as "capacitações" dos indivíduos, referente ao conjunto de funcionamentos existentes naquela sociedade (economia).

A grande dificuldade em operacionalizar um índice de pobreza multidimensional no escopo formulado pela abordagem das capacitações está na definição menos arbitrária de quais seriam as dimensões relevantes para cômputo da pobreza, uma vez que esta é uma decisão que sempre envolve algum grau de arbitrariedade, com implicações diferenciadas quanto aos "beeings and doings" escolhidos pelos indivíduos. Portanto, se a pobreza representa algum tipo de obstáculo ou carência de oportunidade à realização desses funcionamentos, manifestos nos beeings and doings, ela pode ser pensada também como algo que restringe o desenvolvimento econômico-social.

A noção de desenvolvimento, a despeito de suas inúmeras conotações nas ciências sociais aplicadas e mesmo na economia, tem na Declaração do Milênio, celebrada, em setembro de 2000, por 189 Estados Membros das Nações Unidas, uma referência paradigmática assentada nos valores da liber- 
dade, igualdade, solidariedade, tolerância, respeito à natureza e responsabilidade comum (mas diferenciada). Com base nesses valores se definiram os seus oito Objetivos e dezoito Metas, como os elementos norteadores do desenvolvimento a ser perseguido de forma comum por todos os países até o ano de 2015.

Este artigo parte da metodologia do Conjunto Fuzzy, que possibilita o cálculo de indicadores para as $n$ dimensões consideradas relevantes e agregá-las em um único escalar, um indicador-síntese de pobreza multidimensional. Todavia, diferentemente de outros estudos voltados para o Brasil, como Lopes et al. (2004) e Bagolin e Ávila (2006), que também adotam metodologia similar, a contribuição deste artigo é diminuir o grau de arbitrariedade na escolha das dimensões da pobreza a serem consideradas, adotando como referência, para tanto, os objetivos e metas referentes aos Objetivos do Desenvolvimento do Milênio (ONU 2001). Ademais, considerando que as unidades de observação serão os estados brasileiros, os indicadores obtidos não se prendem a uma única base de dado de referência, o que limita o escopo das dimensões da pobreza a serem escolhidos, como em Barros et al. (2006) e Kageyama e Hoffman (2006), ambos tendo restringido suas análises aos dados da Pesquisa Nacional por Amostra de Domicílio - PNAD.

Importante observar que, a despeito da abordagem das capacitações ter como unidade de análise o indivíduo, isto não invalida a possibilidade de se pensar em "funcionamentos e capacitações sociais", isto é, em elementos que sejam objetivos sociais minimamente comuns, como àqueles definidos pelos Objetivos do Milênio, em um contexto mais agregado, como no caso dos estados. Esta é uma informação adicional, pelo menos em um tipo de investigação comparativa entre os estados e regiões do país e que se confronta, por exemplo, àquelas apresentadas pelo IPEA, MP, IPEA (2007), para o Brasil. Ademais, sob a limitação dos dados existentes, a metodologia aqui desenvolvida pode ser empregada para os indivíduos, o que poderia revelar outros tipos de informações úteis, como as desigualdades interestaduais, sem perda de generalidade.

Este artigo, além desta introdução, apresenta-se dividido em mais cinco seções. A segunda descreve os elementos principais da abordagem das capacitações. A terceira discute quais as dimensões humanas relevantes a serem consideradas na elaboração de um índice multidimensional de pobreza. A quarta apresenta a metodologia utilizada para o cálculo do índice multidimensional proposto. A quinta relata os resultados empíricos obtidos, a partir de um exercício para os estados brasileiros no ano de 2002. A última seção apresenta as considerações finais.

\section{A Abordagem das Capacitações}

A Abordagem das Necessidades Básicas é a primeira a reclamar por uma visão humanista, que tenta incorporar valores morais, de justiça, liberdade e dignidade, na avaliação da pobreza. Sob este escopo, esta abordagem procura comparar a situação de cada lugar como uma cesta de bens que expressam necessidades mínimas a serem satisfeitas. A crítica a esta abordagem centra-se no critério de seleção das necessidades e dos limites mínimos a serem estabelecidos (Salama e Destremau 2002).

Em um sentido mais amplo, a abordagem das capacitações difere-se das 
demais abordagens porque compreende um framework normativo para a avaliação individual do bem-estar e de arranjos sociais. Porém, vai além, permitindo também servir de referência para o desenho de políticas e de propostas de mudança social, daí por isso poder ser empregada em um largo campo de aplicações como: economia do bem-estar, política social e filosofia política (Robeyns 2004).

Em alguns aspectos ela é tida como originária das contribuições de Adam Smith, John Stuart Mill e Karl Marx, não obstante seja a partir das contribuições de Amartya Sen e, mais recentemente, de Martha Nussbaum, que ela ganha notoriedade. Como chama atenção Comim et al. (2006), no primeiro caso, a análise de Sen é mais "fechada" na avaliação da fome, pobreza, desigualdade, enquanto que Nussbaum transita na área da filosofia política, convergindo pontos de uma teoria da justiça, com aspectos morais e éticos.

A abordagem das capacitações quebra a noção utilitarista de que os indivíduos são apenas agentes com necessidades, defendendo que são "agentes de mudança", cuja situação de vida (ou bem-estar), depende das oportunidades que lhes são oferecidas, não só como consumidores, mas em todas as esferas sociais. Assim, o bem-estar é mediado pelos funcionamentos e capacitações. Funcionamentos relacionados ao que os indivíduos avaliam ser e fazer e às capacitações como a habilidade dos indivíduos de atingir diferentes combinações de funcionamentos e de permiti-los perseguir seus próprios projetos pessoais de vida (Canova et al. 2005). Sob este aspecto, funcionamentos estariam mais relacionados às condições de vida, uma vez que representariam os diferentes estados (de ser e fazer) que os indivíduos alcançam, enquanto que capacitações estariam mais ligadas à idéia das oportunidades reais que os indivíduos dispõem e que, portanto, definem a liberdade de escolha da vida que eles preferem (Canova et al. 2005, p.10).

Assim, ela representa uma crítica ao utilitarismo, da mesma forma que a Teoria da Justiça de Rawls (1971), ainda que sob diferentes princípios. De fato, enquanto esta última defendia que a comparação interpessoal, sob o propósito da justiça, deveria ser baseada em bens sociais primários, a abordagem das capacitações volta seu foco às dimensões das capacitações ${ }^{1}$, que, inclusive, na visão de um dos seus críticos, Cohen (1996), este conceito, enquanto uma medida de avaliação do bem-estar encontra-se entre as utilidades e os bens primários.

A capacitação de uma pessoa depende de uma variedade de fatores incluindo características pessoais, mas também interligadas ao contexto social. Por essa via, um funcionamento significa a realização de um objetivo, enquanto a capacitação é a habilidade de poder realizar este objetivo. E, portanto, falar em privação de capacitações refere-se à carência de oportunidades para realizar os funcionamentos, uma vez que o "conjunto de capacitações" reflete todo o conjunto de possibilidades que os indivíduos têm para escolher.

A principal distinção analítica da abordagem das capacitações é quanto aos fins e meios do bem-estar e do desenvolvimento, sustentando que, enquanto os primeiros teriam importância intrínseca, os segundos teriam apenas valor instrumental. Sob este ponto de vista, o próprio bem-estar e o desenvolvimento deveriam ser discutidos e avaliados em termos das "capacitações" para os "funcionamentos" (Robeyns 2004).

\footnotetext{
${ }^{1}$ Para uma comparação entre a teoria da justiça de John Rawls e a Teoria das Capacitações ver:
} Robeyns (2004). 
Na idéia original de Sen (2000), o desenvolvimento assume, portanto, o caráter de um processo integrado de expansão das liberdades substantivas (elementares), incluindo aquelas com caráter finalista, isto é, aquelas que concorrem diretamente para a promoção do desenvolvimento, enquanto progresso econômico geral, mas também aquelas que tornam os indivíduos potencialmente mais capacitados a usar suas habilidades individuais, o que lhes confere um valor instrumental, para o alcance dos fins almejados. Desse modo, a liberdade se relaciona a tudo aquilo que está ligado diretamente ao alcance da melhoria das condições de vida da população, assim como às oportunidades que tornam os indivíduos possibilitados de alcançá-las.

Seria pelo conjunto, em um processo de complementação mútua, dos distintos tipos de liberdades instrumentais: i) liberdades políticas; ii) facilidades econômicas; iii) oportunidades sociais; iv) garantias de transparência; e v) segurança protetora, que se levaria a promover a capacidade geral de uma pessoa, enquanto formadoras da liberdade humana em geral.

Assim, como aponta o próprio Sen, o sentido de liberdade se vincula não só aos processos "[...] que permitem liberdade de ações e decisões como às oportunidades reais que as pessoas têm, dadas as suas circunstâncias pessoais e sociais [...]" (Sen 2000, p.31). Daí haver uma vinculação entre as escolhas que os indivíduos podem fazer - "capacitações"- e os funcionamentos que definem essas escolhas possíveis.

No âmbito da abordagem das capacitações proposta originalmente por Amartya Sen, a pobreza está relacionada às restrições que impossibilitam a realização das "capacitações" frente aos funcionamentos que a sociedade interpõe aos seus indivíduos. Assim, pobreza significa privação de capacidades "básicas". Isto expande a noção de pobreza, tal que não fica dependente apenas da renda como geradora de capacitações. É preciso ter sempre em mente que renda se acopla a uma noção instrumental, de modo que a potencialidade da renda tem que ser avaliada considerando sempre a conversão da mesma em funcionamentos.

Assim, como chama atenção (Sen 2000, p.112), o que a perspectiva da capacitação fez para a análise da pobreza foi “[...] melhorar o entendimento da natureza das causas da pobreza e privação desviando a atenção principal dos meios (e de um meio específico que geralmente recebe atenção exclusiva, ou seja, a renda) para os fins que as pessoas têm razão para buscar e, correspondentemente, para as liberdades de poder alcançar esses fins". Desse modo, pode ser que pessoas com diferentes capacitações tenham igual bem-estar, em função do funcionamento realizado.

A pobreza extrema se caracterizaria como uma forma de privação da liberdade econômica, com conseqüências a outras formas de liberdade, tais como: liberdade política e liberdade social. Não obstante, a perspectiva da capacitação serve não como uma teoria para explicar a pobreza, mas uma ferramenta a partir da qual poderia se conceituar e avaliar esse fenômeno (Robeyns 2005).

Em contraste, a riqueza seria aquela situação que, em princípio, leva as liberdades substantivas à condição de plena realização. A questão é quanto à utilidade da riqueza como capaz de tornar possível o que se quer realizar. É por isso que deveria ser encarada apenas como um meio.

Dentro dessa perspectiva, a "abordagem das capacitações" coloca a avaliação do "bem-estar" a partir de duas formas de mensuração diferentes: a primeira considerando os funcionamentos efetivamente realizados (o que uma pessoa fez), a segunda considerando o conjunto "capacitatório" de alternati- 
vas que ela tem (suas oportunidades reais).

Assim, a questão recai sobre a avaliação de como os funcionamentos podem ser atingidos. Por exemplo, se o funcionamento é ter saúde a avaliação recai sobre se existem recursos necessários para esta capacitação como: água potável, saneamento, acesso a médicos, proteção contra infecções e doenças, como vacinação, e conhecimento básico sobre saúde (higiene) que evite doenças. Assim, vários mecanismos de condições de vida que expressem as possibilidades de alcance de cada funcionamento é que são avaliados.

O problema da avaliação recai na questão da heterogeneidade e dos pesos, acerca do que deve ou não ser considerado mais importante ou relevante do ponto de vista do bem-estar individual e coletivo: escolha individual e escolha social. Além do que, volta-se a determinar quais as dimensões relevantes, sob um caráter universal, na tentativa de superar os problemas das diferenças culturais que geram divergências no conceito de qualidade de vida e do próprio bem-estar (Nussbaum e Sen 1996).

Por tudo isso, a abordagem das capacitações, como sugere Robeyns (2005), fornece os próprios fundamentos teóricos do paradigma do desenvolvimento humano, que é o cerne dos Relatórios do Desenvolvimento Humano do Programa das Nações Unidas para o Desenvolvimento - PNUD, e que vem embasar a discussão a respeito dos Objetivos do Desenvolvimento do Milênio. Além do mais, vem ao encontro da noção de desenvolvimento (humano) sustentável, como proposto no Relatório Bruntland (Canova et al. 2005).

\subsection{O problema de operacionalização da abordagem das Capacitações}

Como assinalam Canova et al. (2005), o problema da operacionalização consiste na tradução de conceitos teóricos em empíricos, os quais se convertem em variáveis empíricas úteis nas análises quantitativa e qualitativa. Na abordagem das Capacitações de Sen, ainda, segundo esses autores, isto consiste na tradução de recursos em "beings and doings" que sejam valorados, a partir do qual as várias combinações de funcionamentos potencialmente "atingíveis" poderiam ser escolhidas (estas possibilidades de escolhas é que formam o espaço de capacitações).

Esses mesmos autores sustentam que, para tornar operacional a abordagem das Capacitações de Sen, seria necessário restringir o modelo ao vetor de escolhas de funcionamentos, o que evitaria os problemas relacionados à mensuração das capacitações e superaria o problema da não observação. Além disso, isto por si só já poderia ser visto como uma avaliação elementar do conjunto de capacitações.

É preciso se ter claro o fato de que, na avaliação dos funcionamentos ou na falta deles, toma-se uma medida considerada como geral, indistinta das capacitações. Isto porque na avaliação das capacitações está se levando em conta os "funcionamentos que uma pessoa poderia ter atingido", o que é de natureza contrafactual, uma vez que a pessoa pode ter escolhido um "nãofuncionamento" por algum motivo.

Nesse sentido é que, na abordagem das capacitações, a renda, bem como sua transformação em bens e serviços, apenas permite alcançar funcionamentos. Assim, pessoas desejam bens e serviços porque permitem o alcance de funcionamentos e devem ser avaliadas como tal, isto é, meios. Além do mais, por mais que existam esses meios, o real valor dos funcionamentos para os indivíduos depende dos chamados fatores de conversão, que, para Robeyns 
(2004), são três: i) características pessoais: metabolismo físico, condição física, sexo, inteligência entre outros; ii) características sociais: políticas públicas, normas sociais, práticas discriminatórias, hierarquia social, papel dos sexos na sociedade, estrutura de poder, entre outras; iii) características ambientais: clima, infra-estrutura, instituições, bens públicos entre outras.

\section{Quais as Dimensões Humanas Relevantes}

Uma primeira discussão no sentido da definição de um indicador multidimensional diz respeito à tentativa de responder quais as dimensões relevantes a serem consideradas para dar conta do desenvolvimento humano. Aspectos relacionados ao bem-estar, em uma visão intrinsecamente utilitarista; ou apenas elementos relacionados às necessidades básicas; ou, ainda, valores humanos universais como justiça, liberdade, dignidade entre outros entram no rol das escolhas possíveis.

Alkire (2002) estabelece quatro razões acerca da necessidade de se escolher dimensões. A primeira vem ao encontro de fundamentar empírica e epistemologicamente o objetivo multidimensional do desenvolvimento humano e, por essa via, da pobreza multidimensional como algo teórico e empiricamente defensável. A segunda, de natureza prática, relaciona-se à necessidade por efetivas metodologias para as comunidades avaliarem tradeoffs. A terceira razão porque ajuda grupos a identificar impactos não intencionais. A quarta está relacionada ao fato de que teorias que não são "amigáveis" ao usuário não se difundam.

Raws, Sen e Nussbaum têm diferentes perspectivas de quais seriam as dimensões mais relevantes a considerar, cada qual sob um viés mais específico de determinados elementos.

Rawls (1971) define os aspectos mais importantes do bem-estar relacionados a cinco grupos de bens sociais primários: a) direitos básicos e liberdades; b) liberdade de movimento e escolha de ocupação; c) poderes e prerrogativas de comando e posições de autoridade e responsabilidade; d) renda e riqueza; e e) bases sociais do auto-respeito ${ }^{2}$.

Nussbaum, sob a influência das "virtudes não-relativas", do homem, segundo Aristóteles, enumera 11 capacitações humanas funcionais centrais (Nussbaum apud ALKIRE, 2001 ): i) vida - ser capaz ter uma vida com uma longevidade normal, o que pressupõe não morrer prematuramente ou não ser reduzida quando não valha a pena morrer; ii) saúde corporal - ser capaz de ter boa saúde, incluindo a saúde reprodutiva, o que pressupõe ser adequadamente nutrido e ser adequadamente abrigado; iii) integridade corporal - no sentido do indivíduo ser capaz de se deslocar livremente, o que implica a proteção contra qualquer tipo de violência que restrinja esta mobilidade e, ao mesmo tempo, forneça aos indivíduos oportunidades para a sua satisfação sexual e escolhas em matéria de reprodução; iv) sentidos, imaginação e pensamentos ser capaz de usar seus sentidos, imaginação e razão de forma verdadeiramente

\footnotetext{
2 Esta lista de bens primários está relacionada diretamente aos dois princípios fundamentais definidos por Rawls como norteadores da justiça na sociedade: 1 . cada pessoa deve ter o mesmo direito a um completo esquema de liberdades fundamentais, compatível com um esquema similar para todos; 2. desigualdades sociais e econômicas são para satisfazer duas condições: primeiro, elas existem para alcançar posições abertas para todos sob condições de igualdade de oportunidades; segundo, elas devem fornecer maiores benefícios aos indivíduos com menores vantagens na sociedade (menos favorecidos).
} 
humana. Isto inclui oportunidades de educação através da alfabetização, matemática básica e treinamento científico, como também a possibilidade de usar seus sentidos, imaginação e razão para se auto-expressar, e fazer suas próprias escolhas em matéria de religião, artes, entre outros; v) emoções - ser capaz de expressar suas emoções, reforçando àquelas que lhes são boas e evitando aquelas que não lhes são; vi) razão prática - o que resulta em ser capaz de formar uma concepção do bem e ter uma clara reflexão sobre o planejamento de sua própria vida; vii) afiliação - ser capaz de viver por e para os outros, o que implica uma capacitação para justiça, amizade e se envolver com os problemas dos outros. Neste particular, deveria ser cultivado um sentimento de igualdade, rejeitando qualquer tipo de descriminação de sexo, raça, religião, etnia e origem nacional; viii) Outras espécies - ser capaz de respeitar animais, plantas, enfim, respeitar as outras espécies que formam a natureza; ix) jogar - ser capaz de rir, e de se engajar em atividades ligadas ao entretenimento e lazer; $x$ ) controle sobre o seu próprio ambiente - ser capaz de participar efetivamente de decisões políticas, que influenciam sobre sua própria vida; xi) material - ter direito a propriedade, não apenas formalmente, mas também em termos de oportunidades. Isto inclui ter direito à propriedade sob bases iguais aos outros indivíduos, e direito a procurar emprego em condição de igualdade aos demais.

Sen (2000) identifica não propriamente quais seriam as dimensões mais importantes, mas cinco fontes distintas de diversidade humana, que influenciariam diretamente nas escolhas dessas dimensões: i) heterogeneidades pessoais: características relacionadas aos atributos físicos das pessoas: idade, sexo, raça, entre outros; ii) diversidades ambientais, que versa sobre as características do meio ambiente dos estados como: clima, diferenças geográficas entre outras; iii) variações no clima social: influências do ambiente social, que influencia na qualidade de vida das pessoas; iv) diferenças de perspectivas relativas: influenciadas neste caso pelos elementos de natureza cultural como hábitos e costumes e valores; v) distribuição das famílias: entendido como unidade básica de consumo e de bem-estar.

Embora haja pontos convergentes entre os três autores, percebe-se que o escopo analítico da escolha das dimensões possuem condicionantes individuais, sociais e mesmo ambientais (espaciais), que tornam a escolha das dimensões algo a princípio que não poderia ser determinado de forma padrão, a não ser algo que seja minimamente consensual, com relação aos funcionamentos mínimos que deveriam ser realizados por todos. Este tem sido, de uma forma ou de outra, o caminho tortuoso seguido pelas Nações Unidas, a partir do Programa das Nações Unidas para o Desenvolvimento - PNUD, na tentativa de se criar medidas e índices, que reflitam o desenvolvimento humano e permitam a sua evolução ao longo do tempo. Os Objetivos do Desenvolvimento do Milênio em parte vêm na perspectiva de criar um senso comum entre os países nessa direção.

\subsection{Opção pelos "Objetivos do Milênio"}

A Declaração do Milênio, em 2000, pactuada como os grandes objetivos (metas) a serem perseguidos pelos 189 Estados-membros da Assembléia Geral das Nações Unidas, na promoção do Desenvolvimento Econômico, serviu como a referência para a escolha das dimensões possíveis que comporiam o indicador de pobreza multidimensional perseguido. Assim, essa referência, ao mesmo 
tempo em que diminuiria o grau de arbitrariedade atribuído à escolha das dimensões, serviria como parâmetro de avaliação do esforço de busca do desenvolvimento humano promovido pelos estados brasileiros na tentativa de alcançar os oito objetivos e dezoito metas, constantes naquela declaração.

Mesmo admitindo que se mantenha um certo grau de arbitrariedade na escolha das dimensões ancoradas nos Objetivos do Desenvolvimento do Milênio, todavia alivia-se outro problema relacionado ao caso brasileiro ${ }^{3}$ : a restrição da opção da escolha das dimensões a partir de dados cuja base de observação são indivíduos ou famílias. De fato, como as unidades de referência não são as mesmas para a PNAD, POF etc., que entram na amostra, o grau de possibilidades fica limitado se a agregação for feita a partir das características desses indivíduos, famílias ou domicílios pesquisados.

Além do mais, como bem destacado pelo Relatório do Desenvolvimento Humano de 2003, do PNUD, dedicado exclusivamente aos objetivos do Desenvolvimento do Milênio, estes têm uma vinculação direta com as capacidades fundamentais que promovem o desenvolvimento humano: i) viver uma vida longa e saudável; ii) ser instruído; iii) ter um nível de vida digno e suas condições para tanto (funcionamentos): i) sustentabilidade ambiental; ii) equidade, em particular de gênero; iii) facilitar o ambiente econômico mundial.

A Tabela 1 apresenta os Objetivos e Metas do Desenvolvimento do Milênio e os indicadores a serem utilizados para compor o índice de pobreza multidimensional, usando para tanto o recurso metodológico de torná-los detrimental (prejudicial), isto é, considerá-los em termos de uma restrição para se alcançar cada objeto e meta e, portanto, de se atingir o funcionamento associado ao objetivo e meta estabelecidos.

\section{A Metodologia Utilizada}

Como já fora assinalado por vários autores, entre eles Sen (1976), o primeiro passo para construção de um indicador de pobreza, seja ele unidimensional ou multidimensional, envolve dois níveis. O primeiro é a identificação, isto é, qual o atributo ou os atributos que permite $(\mathrm{m})$ apontar determinados indivíduos como pertencentes ao conjunto dos indivíduos pobres, o que fora definido na seção anterior. O segundo é a agregação.

Como chama atenção Comim et al. (2006), existem dois níveis do desenvolvimento humano para se levar em conta em tal índice se dar conta em um índice para tal. Um relacionado à formação das capacitações humanas, estando relacionados aos níveis de saúde e educação, por exemplo. E outro vinculado a como essas capacitações são utilizadas.

No contexto deste estudo será adotada a metodologia derivada da chamada Teoria de Fuzzy Sets, cujo diferencial em relação a outras metodologias é possibilitar o cálculo de cada atributo em separado (no caso dimensões) e cada unidade de análise e depois poder agregá-los em um índice síntese ${ }^{4}$

Segundo Zadeh (1965) apud Klir e Yuan (1995), conjuntos fuzzy, são conjuntos cujos limites não são precisos, de modo que a pertinência em relação a este conjunto fuzzy não é estabelecida em matéria de afirmação ou negação,

\footnotetext{
${ }^{3}$ Mediada, em muitos casos, como chama a atenção Barros et al. (2006), pelo pragmatismo da disponibilidade dos dados.

4 Para uma justificativa metodológica mais rigorosa, ver Cheli e Lemmi (1995); Lopes (2003); Verkuilen (2005).
} 





mas em matéria de grau. Em matéria de lógica isto significa que quando $A$ é um conjunto fuzzy e $x$ é um objeto relevante, a proposição " $x$ é um membro de $A$ " não é necessariamente verdadeira ou falsa, mas pode ser verdadeira somente para algum grau, o grau para o qual " $x$ é atualmente um membro de $A^{\prime \prime}$. Neste sentido, é mais comum, mas não requerido, expressar graus de pertinência em um conjunto fuzzy, como graus de verdade das proposições associadas pelos números no intervalo fechado [0,1] (Klir e Yuan 1995).

Os valores extremos neste intervalo, 0 e 1, representam, então, respectivamente, a negação e afirmação total de pertinência em um dado conjunto fuzzy, tanto quanto a falsidade e verdade da proposição associada. Por outro lado, os outros indivíduos que caem dentro do intervalo, mas não aos seus extremos, pertencem ao conjunto fuzzy a um maior ou menor grau como indicado por um maior ou menor grau de pertinência, geralmente representados por valores de números reais variando no intervalo aberto entre 0 e 1 . A função que associa valores reais que indicam o grau de pertinência dos elementos do conjunto fuzzy é denominada de "função de pertinência".

Mais formalmente a função de pertinência de um conjunto fuzzy A é denotada por $\mu_{A}$, tal que:

$$
\mu_{A}: X \rightarrow[0,1]
$$

Desse modo, dada uma população $A=\left\{a_{1}, \ldots, a_{i}, \ldots, a_{n}\right\}$ composta por $n$ estados, considerar-se-á como estados pobres aqueles apresentam algum grau de pobreza em pelo menos algum dos $m$ atributos de $X$. Estas famílias vêm a constituir um subconjunto $B$ de $A$.

O grau de intensidade da pobreza $x_{i j}$ do $i$-ésimo estado $(i=1, \ldots, 27)$ referente ao $j$-ésimo atributo $(j=1, \ldots, m)$ para of fuzzy set $B$ é dado, como proposto originalmente por Cheli e Lemmi (1995):

$$
\mu=\left[X_{j}\left(a_{i}\right)\right]=x_{i j}, 0 \leq x_{i j} \leq 1
$$

De modo que $x_{i j}=1$, se o $i$-ésimo estado é dotado do $j$-ésimo atributo; $x_{i j}=0$, se o $i$-ésimo estado não é dotado do $j$-ésimo atributo; $0<x_{i j}<1$, se o $i$-ésimo estado é dotado do j-ésimo atributo em algum grau entre 0 e 1.

$O$ índice de pobreza do $i$-ésimo estado, ou seja, o grau de intensidade da pobreza da $i$-ésimo estado para o conjunto $f u z z y B$, seria, então, definido como a média ponderada dos atributos $x_{i j}$,

$$
\mu_{B}\left(a_{i}\right)=\sum_{j=1}^{m} x_{i j} w_{j} / \sum_{j=1}^{m} w_{j},
$$

onde o peso do j-ésimo atributo é determinado pela função de ponderação

$$
w_{j}=\ln \left[n / n-\sum_{i=1}^{n} x_{i j}\right] \geq 0,
$$

A principal motivação para a utilização da função de ponderação (3) reside no fato de que os pesos por ela determinados são tais que "quanto mais pobre for o conjunto dos estados em relação a um determinado atributo, maior peso terá este atributo no cálculo ponderado do índice de pobreza de cada estado", de modo que, quanto mais próxima de zero a soma $\sum_{i=1}^{n} x_{i j}$ dos graus de intensidade de pobreza de todos os estados em relação a um j-ésimo atributo 
estiver, mais próximo de zero será o valor do peso $w_{j}$, enquanto que, quanto mais próxima de $n$ a soma estiver, maior será o valor de $w_{j}$.

$\mathrm{O}$ índice de pobreza $\mu_{B}\left(a_{i}\right)$ mede, portanto, o grau de pobreza do $i$-ésimo estado como uma função ponderada dos $m$ de atributos. Esta é uma medida do grau de exclusão social, de privação relativa e de não-dotação de algumas capacitações relativas ao $i$-ésimo estado, privação no sentido de poder gozar de suas capacitações, no sentido dado por Sen.

O que indica que quanto maior o índice de pobreza, ou seja, quanto mais próximo de 1, maior a pobreza relativa da população em análise.

A segunda parte da metodologia adotada segue em parte a definida em Lopes (2003), Bagolin e Ávila (2006) e Picolloto et al. (2007), que corresponde à construção da função pertinência correspondente a cada dimensão considerada, isto é, à construção de diferentes matrizes para a definição da função linear que atribuem valores a serem considerados por essa função em uma escala de 0 a 1 , como requer a metodologia do conjunto fuzzy.

\subsection{A definição das funções pertinências}

Foram considerados doze indicadores, conforme aqueles recomendados pela ONU e dispostos no Quadro 1. A construção da matriz pertinência foi desenvolvida em duas etapas. A primeira fornece a informação do estado em relação a ele próprio, enquanto direcionado a alcançar a meta dos objetivos do milênio ligada àquela dimensão da pobreza em particular - equação (4). A segunda normaliza a evolução do estado em relação aos demais estados, tal que a intensidade da pobreza fica restrita a algum grau entre 0 e 1 - equação (5). Assim, pode-se entender o índice de pobreza final, o indicador-síntese de pobreza multidimensional, como carregando consigo tanto um caráter absoluto, quanto relativo.

$$
\tilde{x}=\frac{x_{i ; t}-x_{\text {meta } i} \text {; ano base }}{x_{i ; \text { ano base }}-x_{\text {meta } i \text {; ano base }}}
$$

No que o ano base é a data de referência para o qual foi traçada a meta para 2015. No caso específico deste estudo foi adotado o ano de 1992.

$$
\mu=\frac{\tilde{x}-\tilde{x}_{\min }}{\tilde{x}_{\max }-\tilde{x}_{\min }}
$$

Para efeito de ilustração do significado do índice, como definido acima, apresenta-se a seguir uma situação em que este é simulado para três estados (unidades da Federação) $A, B$ e $C$, comparando-os com se fosse uma corrida ${ }^{5}$.

Assim, suponha que a meta a ser alcançada em 2015 seja a metade do valor daquele indicador para o ano de 1992 - o ano base - e que o ano t do índice a ser calculado seja o ano de 2002.

\begin{tabular}{cccc}
\hline Estado & $X(1992)$ & $X / 2(1992)$ & $X(2002)$ \\
\hline$A$ & 0,6 & 0,3 & 0,42 \\
$B$ & 0,5 & 0,25 & 0,3 \\
$C$ & 0,8 & 0,4 & 0,72 \\
\hline
\end{tabular}

Neste caso, a Figura 1 representaria a corrida de cada estado pela obtenção do objetivo:

\footnotetext{
${ }^{5}$ Nestes termos, cada estado é um corredor.
} 
$(0.19,0.39)$

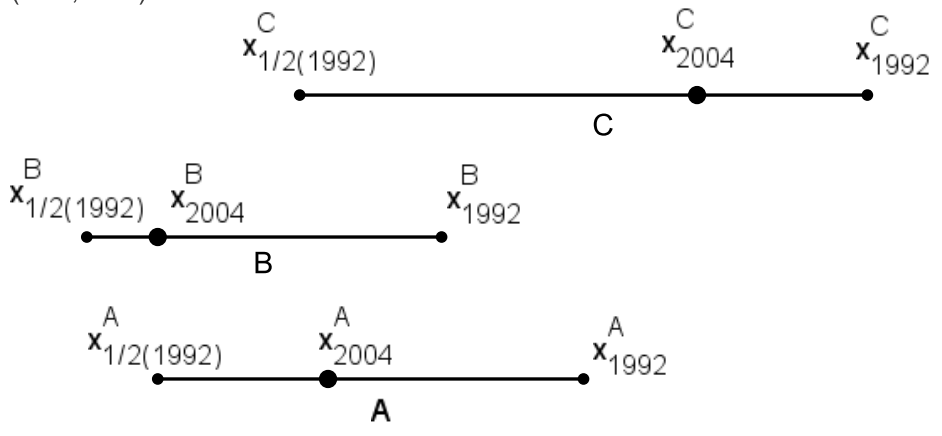

\begin{tabular}{llllllll}
\hline 0.2 & 0.3 & 0.4 & 0.5 & 0.6 & 0.7 & 0.8 & 0.9
\end{tabular}

$(0.94,-0.12)$

Figura 1: Representação gráfica da primeira etapa do índice proposto Posição absoluta.

A primeira etapa do índice proposto (equação 4) retrata o posicionamento de cada corredor (estado) relativamente à sua própria corrida. A obtenção do valor 0 para o índice representaria a chegada do estado no objetivo traçado para o indicador em questão, enquanto que a obtenção do valor 1 representaria que o estado não obteve melhora alguma. $\mathrm{O}$ valor do índice representa exatamente o percentual da distância que o estado ainda tem a percorrer. Por exemplo, o índice para o Estado A seria:

$$
\mu_{A}=\frac{0.42-0.3}{0.6-0.3}=0.4
$$

que significa que ainda resta $40 \%$ do trabalho a ser realizado para o alcance do objetivo proposto. Observe que a eventual obtenção de um valor negativo para o índice, significaria, em termos percentuais, relativamente à melhoria proposta, a superação da meta estabelecida. O gráfico abaixo retrata os índices obtidos pelos três estados e serve bem à comparação entre nossos corredores:

A segunda parte do índice proposto retrata ainda a situação da corrida pelo alcance da meta, mas desta vez relativamente ao desempenho dos demais corredores. A obtenção do valor 0 para este índice por um estado significa que se trata do estado em melhor situação dentre os demais na corrida ou que se trata de um estado dentre os que já alcançaram a meta estabelecida (situação ótima), enquanto que a obtenção do valor 1 diria que se trata do estado que está mais atrás no alcance da meta. A representação gráfica deste índice seria homotética à representação obtida do intervalo entre o menor e o maior valor no índice anterior. $\mathrm{O}$ estado que obtivera menor valor no índice anterior (B, no caso), agora obtém índice 0 , enquanto que o estado de maior índice anterior obtém índice 1 . Os demais estados obtêm índices entre 0 e 1 , proporcionais aos valores que obtiveram no índice anterior, relativamente ao pior e ao melhor desempenho a pouco aludidos. Por exemplo, o estado A teria por índice: 




Figura 2: Representação gráfica da primeira etapa do índice proposto - Posição relativa.



Figura 3: Representação gráfica da primeira etapa do índice proposto - Simetria entre a posição Absoluta e Relativa.

$$
\eta_{A}=\frac{0.4-0.2}{0.8-0.2}=0.333 \ldots
$$

A Figura 3 ilustra a atribuição deste outro índice, comparando-o com os valores obtidos no índice anterior:

Esta segunda parte do índice proposto possui a vantagem de indicar precisamente a situação do estado para aquele indicador, dentro do espectro da totalidade dos estados (por exemplo, o fato de $\eta_{A}=0.333 \ldots$ indica que a progressão para o alcance da meta pelo estado A está bem mais próxima da progressão feita pelo estado de melhor desempenho do que do de pior desempenho), mas nada diz acerca de, por exemplo, quanto falta ao estado A para o alcance de sua meta. A primeira parte do índice fornece este dado precisamente, mas é incapaz de posicionar o estado em relação aos outros (saber apenas que $\mu_{A}=0.4$ nada diz acerca de como o estado A se encontra face aos demais estados).

A Função Pertinência ficaria, portanto, definida como:

$$
\frac{\tilde{x}_{i}-\tilde{x}_{i, \min }}{\tilde{x}_{i, \max }-\tilde{x}_{i, \min }}
$$


em que

$$
\tilde{x}_{i}= \begin{cases}0 & \text { se } x_{i ; t} \leq x_{\text {meta } i} \\ \frac{x_{i, t}-x_{\text {meta }} i}{x_{i ;(\text { ano base })^{-} x_{\text {meta } i}}} & \text { se } x_{i ; t}>x_{\text {meta } i} \\ 1 & \text { se } \tilde{x}_{i}=\tilde{x}_{i, \max }\end{cases}
$$

\section{RESULTADOS EMPÍRICOS}

\subsection{Dos dados}

São muitas as bases de dados utilizadas referentes às variáveis utilizadas para compor cada indicador referente aos objetivos e metas dos Objetivos do Desenvolvimento do Milênio. Para dar maior operacionalidade e permitir uma comparação com outros estudos, que já vêm sendo realizados no âmbito dos Objetivos do Milênio, os indicadores utilizados se assemelham aos adotados em IPEA, SPI/MP (2007) e IDHS-PUC MINAS, PNUD Brasil (2007).

Com relação ao Objetivo 1, as informações referentes aos indicadores da meta 1: proporção de indigentes e hiato de indigência referem-se à base IPEADATA do Instituto de Pesquisa Econômica Aplicada - IPEA ${ }^{6}$. Para o objetivo 2, e indicadores da meta 3: proporção de analfabetos de 15 a 24 anos e defasagem escolar, foram utilizados dados do censo escolar (2002) do Instituto Nacional de Estudos e Pesquisas Educacionais - INEP.

Quanto aos indicadores da meta 4: déficit na igualdade de candidatos eleitos dos gêneros masculino e feminino, foram utilizadas informações constantes no IPEADATA.

Indicadores referentes à Meta 5; taxa de mortalidade de crianças menores de 5 anos e taxa de mortalidade infantil; Meta 6: taxa de mortalidade materna; Meta 7: taxa de Incidência de HIV/Aids, todos tiveram como fonte, o banco de dados - DataSUS, do Ministério da Saúde ${ }^{7}$.

Para o Objetivo 7, foram obtidos informações relativas apenas dos indicadores da Meta 10: déficit da população com acesso a água potável e déficit da população com acesso ao esgotamento sanitário a partir do IPEADATA, com referência nos dados da Pesquisa Nacional de Amostra por Domicílio - PNAD do IBGE.

\subsection{Os resultados empíricos para os indicadores}

Considerando como os estados evoluíram com relação ao alcance de cada Objetivo do Milênio, observa-se pela Tabela 1, que alguns indicadores que descrevem o comportamento das metas imputadas aos Objetivos 1, 4, e 7 apresentaram-se negativos, denotando que para alguns estados houve um crescimento acima da meta entre os anos de 1992 e 2002. São representativos os casos dos estados do Paraná e Santa Catarina que tiveram um crescimento de $38 \%$ e $44 \%$ acima das suas respectivas médias, quanto ao hiato médio de indigência entre os dois anos considerados.

Quanto aos Objetivos 6 e 7, o estado de São Paulo teve um desempenho acima da meta para os indicadores: taxa de incidência de Aids e déficit do abastecimento de água, em $53 \%$ e $45 \%$, respectivamente.

\footnotetext{
${ }^{6}$ Disponível em http://www.ipeadata.gov.br

${ }^{7}$ Disponível em http://w3.datasus.gov.br/datasus.
} 
É o déficit no abastecimento de água que, no conjunto dos estados, apresenta um melhor desempenho entre todos os indicadores considerados, com 15 estados ou cerca de 55\% destes apresentando uma evolução acima das metas dos estados entre o ano de 1992 e 2002. O Distrito Federal e os estados de Santa Catarina e Roraima representam os casos extremos de alcance e superação das metas relativos a este indicador específico para o Objetivo 7 .

No outro extremo, para o conjunto dos estados, os Objetivos 4 e 5, referentes aos indicadores de redução da taxa de mortalidade de crianças abaixo de cinco anos e taxa de mortalidade materna, foram os menos exitosos quanto à evolução em direção ao alcance das respectivas metas para os estados. Assim, em ambos os casos, nove estados (33\%) teriam de percorrer uma distância de mais de $100 \%$ para a obtenção das metas perseguidas, considerando o ano de 2002.

Do ponto de vista espacial, não existe uma diferença bem definida quanto às macroregiões do país, relativa ao grau de eficiência no alcance das metas dos Objetivos Milênio, quando se ordena todos os estados relativos às suas metas respectivas.

Não obstante, quando se toma a média dos resultados para os estados por região, os indicadores utilizados apontam os estados da Região Nordeste e Região Norte como aqueles que apresentaram maiores distâncias para o cumprimento das suas metas. Assim, por exemplo, em média, os estados da Região Nordeste, seguidos de perto pelos estados da Região Norte, apresentaram maior dificuldade no cumprimento das metas relativas aos indicadores que representam o Objetivo 2.

A meta de redução da mortalidade materna foi aquela que apresentou uma dificuldade de ser cumprida mais homogeneamente distribuída entre as regiões brasileiras sendo, entretanto, mais desfavorável sua evolução para aqueles estados que compõem a Região Sul.

Por sua vez, a meta de reversão da tendência da taxa de incidência de AIDS, relativa ao Objetivo 6, é também uma meta que apresenta grande dificuldade de ser cumprida entre dois extremos do país, com os estados da Região Norte e Região Sul, com médias muito elevadas acima de 400\%.

Por outro lado, quanto aos estados que apresentaram uma menor eficiência para o alcance dos Objetivos, destaca-se o comportamento do estado de Roraima, que ainda precisa crescer em mais de $100 \%$ em relação aos indicadores, que representam as metas dos Objetivos 1 e 5. Especificamente com relação aos indicadores das metas do Objetivo 1, esta tarefa demandará um esforço em mais de $400 \%$. Além disso, este estado apresenta uma média da evolução dos indicadores relativos ao Objetivo 2 que também o distancia em mais de $100 \%$, em relação à superação desse Objetivo, na comparação do quanto evoluiu a média desses indicadores entre os anos de 1992 e 2002.

Uma vez que os estados no ano de 1992 estavam em condições bastante diferenciadas quanto ao cumprimento de suas metas, o que os colocava em condições diferenciadas nas posições de largada da "corrida" em busca do alcance das mesmas, é apresentado na Tabela 3 o ordenamento dos estados por indicador pertinente às metas dos Objetivos do Milênio, com a diferença que o interesse agora é quanto à posição relativa dos estados frente aos demais estados. Assim, no numerador está expressa a distância para o cumprimento da média no ano de 2002, adotando que para os casos em que o estado já tenha atingido a meta estabelecida o índice é zero. E no denominador encontra-se a distância do estado de pior desempenho para o alcance da sua meta, de modo 


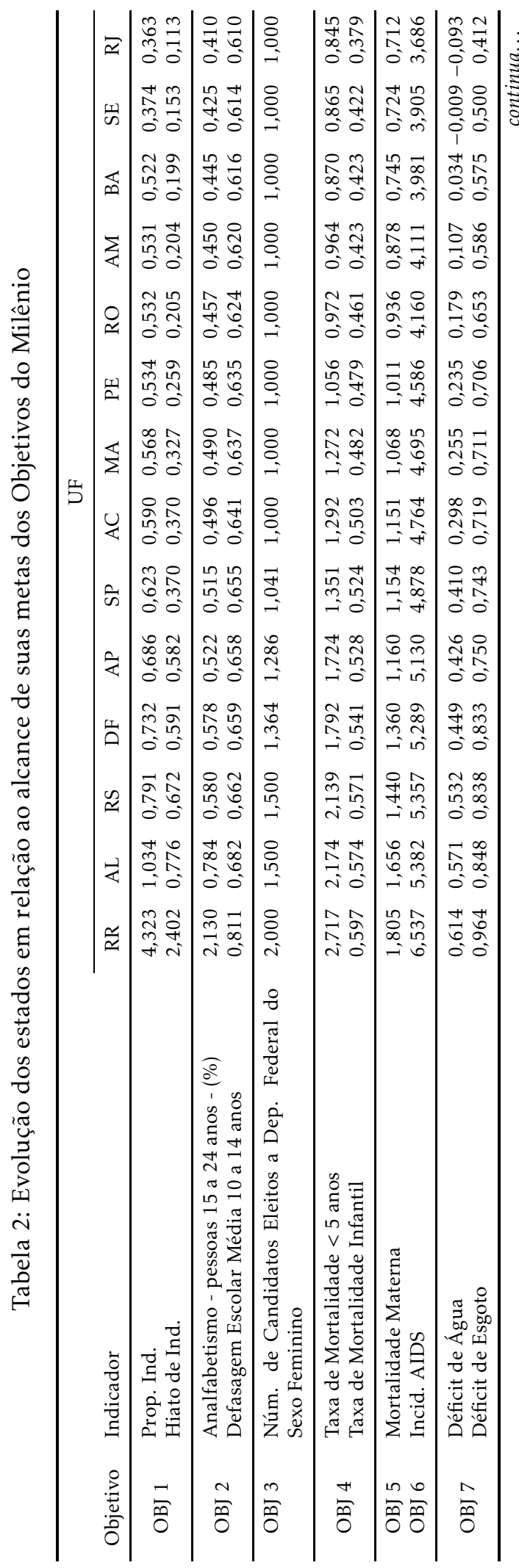







que neste caso o estado em questão apresente índice 1, ficando a comparação de todos os demais estados em relação a ele.

Levando em consideração essa formulação, naturalmente os piores estados no cumprimento de suas metas respectivas são os mesmos já evidenciados na análise anterior. Ao mesmo tempo, e também ao encontro da análise anterior na Tabela 2, não existe uma distribuição espacial bem definida entre aos estados quanto às primeiras posições, e, portanto, quanto à distribuição do grau de ineficiência dos estados relativo ao pior estado em cada caso.

Não obstante, é interessante observar que entre os estados que não cumpriram suas metas, para alguns indicadores, relativos às metas do Objetivo 6 - Sustentabilidade Ambiental; Objetivo 4 - Reduzir a Mortalidade Infantil e Objetivo 5 - Diminuir a Mortalidade Materna, os valores relativos são altos, vis a vis os valores relativos dos índices que representam as metas do Objetivo 2 - Atingir o Ensino Básico Universal, o que denota que, nesse segundo caso, a dispersão foi proporcionalmente maior no alcance das metas específicas a esse objetivo, em parte sendo atribuído ao desempenho mais desproporcional dos estados da Região Norte e Nordeste.

Considerando o índice multidimensional sintético para os Objetivos do Milênio (Tabela 4), observa-se que entre os piores desempenhos estão os estados das regiões Norte e Nordeste, com cerca de nove estados pertencentes a estas entre as dez piores colocações.

Situação mais extremada é a do estado do Rio Grande do Sul, sendo o único estado das regiões Sul e Sudeste que se situa no extremo inferior da distribuição. Importante mencionar que contribuíram de forma mais significativa para a perfôrmance, por assim dizer, negativa do estado do Rio Grande do Sul, os indicadores relativos aos Objetivos 1: proporção de indigentes e hiato de indigência; Objetivo 4: taxa de mortalidade de menores de 5 anos e taxa de mortalidade infantil e Objetivo 5: Mortalidade Materna.

Por sua vez, no outro extremo está o estado de São Paulo, que pode ser dito o mais eficiente em termos gerais, quanto ao cumprimento dos Objetivos do Milênio e, portanto, com menor índice de pobreza multidimensional. Sua perfôrmance pode ser atribuída, principalmente, com relação aos seguintes a evolução dos Objetivos do Milênio: Objetivo 2, especialmente, na melhoria da defasagem escolar média; Objetivo 6, queda taxa de incidência de AIDS; e Objetivo 7, quanto à diminuição dos déficits de esgoto e de água. Todavia, a despeito deste fato, observa-se uma dispersão pequena do índice, com desviopadrão de 0,115 para um índice médio de cerca de 0,428.

Vale mencionar, entretanto, alguns casos particulares, como os dos estados do Ceará, Sergipe e, principalmente, Rio Grande do Norte, que se encontram no terço inferior da distribuição, com índices variando entre 0,284 e 0,372 e do estado do Rio de Janeiro, que encontra-se próximo ao valor mediano da distribuição.

Entre os fatores que mais contribuíram para o bom desempenho relativo dos estados do Ceará, Sergipe e Rio Grande do Norte estão a melhora relativa dos Objetivos 1: proporção de indigentes e hiato de indigência; Objetivo 2: Analfabetismo entre pessoas de 15 a 24 anos e Defasagem Escolar Média de 10 a 14 anos; Objetivo 3: Número de Candidatos Eleitos Deputados do Sexo Feminino; Objetivo: Taxa de Mortalidade de Menores de 5 anos e Taxa de Mortalidade Infantil e Objetivo 5: Incidência de Aids.

Quanto ao desempenho do Rio de Janeiro, os fatores que mais contribuíram para o seu fraco desempenho relativo foram: Objetivo 1, Proporção de 










Tabela 4: Índice de pobreza multidimensional a partir dos Objetivos do Milênio.

\begin{tabular}{lc}
\hline \multicolumn{1}{c}{ UF } & $\begin{array}{c}\text { Índice de pobreza } \\
\text { multidimensional }\end{array}$ \\
\hline Rio Grande do Sul & 0,584 \\
Amazonas & 0,576 \\
Acre & 0,567 \\
Rondônia & 0,565 \\
Mato Grosso do Sul & 0,558 \\
Piauí & 0,528 \\
Pará & 0,516 \\
Tocantins & 0,506 \\
Alagoas & 0,502 \\
Pernambuco & 0,486 \\
Maranhão & 0,478 \\
Amapá & 0,473 \\
Goiás & 0,471 \\
Mato Grosso & 0,459 \\
Rio de Janeiro & 0,432 \\
Roraima & 0,414 \\
Bahia & 0,405 \\
Paraíba & 0,398 \\
Ceará & 0,372 \\
Santa Catarina & 0,367 \\
Sergipe & 0,341 \\
Espírito Santo & 0,296 \\
Paraná & 0,290 \\
RIO Grande do Norte & 0,284 \\
Minas Gerais & 0,265 \\
Distrito Federal & 0,249 \\
São Paulo & 0,164 \\
\hline Obs no computo do & \\
\hline
\end{tabular}

Obs: no cômputo do índice acima, observam-se os seguintes pesos obtidos para cada dimensão: prop. Ind.: 0,18; hiato de ind.: 0,23; analf. 15 a 24 anos: 0,15; def. escolar: 0,49; cand. dep. fem: 0,41; tx. mort $<5$ anos: 0,48 ; tx. mort. inf.: 0,67 ; mort. mat.: 0,56; inc. AIDS: 0,59; def. água: 0,44; déf. san: 1,00 Fonte: Elaboração dos autores. 
Tabela 5: Correlação entre o IPM e algumas variáveis selecionadas

\begin{tabular}{lccccc}
\hline & IPM & PIB & GAST SOC. & TRANSF. & INVEST. \\
\hline IPM & 1,00 & $-0,312$ & $-0,335$ & $-0,388$ & $-0,234$ \\
PIB & $-0,312$ & 1,00 & 0,996 & 0,754 & 0,718 \\
GAST. SOC. & $-0,335$ & 0,996 & 1,00 & 0,798 & 0,7527 \\
TRANSF. & $-0,388$ & 0,755 & 0,798 & 1,00 & 0,689 \\
INVEST. & $-0,234$ & 0,718 & 0,752 & 0,689 & 1,00 \\
\hline
\end{tabular}

Fonte: Tesouro Nacional; IPEADATA.

Indigentes e Hiato de Indigência; Objetivo 4, com elevadas taxas de mortalidade de menores de 5 anos e mortalidade infantil e Objetivo 5, Mortalidade Materna.

Por fim, é interessante estabelecer uma correlação entre os resultados obtidos para os estados quanto ao indicador de pobreza multidimensional calculado e algumas variáveis que podem ter influência sobre o seu desempenho. Assim, na Tabela 5, pode-se visualizar uma correlação negativa entre o Índice de Pobreza Muldimensional - IPM, o tamanho das economias dos estados medida pelo PIB, o volume dos gastos sociais em educação, habitação, saneamento, transporte, previdência social, as transferências federais (correntes e de capital) e os investimentos realizados nos estados em cada caso.

É de se esperar que o aumento das transferências federais para os estados, aumentando sua capacidade de investimento e a transformação desses recursos em ações nas diversas esferas sociais, a partir do gasto público, possam contribuir para que estes estados possam melhorar os indicadores sociais, não obstante problemas de focalização das políticas públicas, entre outros, poderiam diminuir a eficiência alocativa desses recursos.

Assim, explica-se porque os resultados da Tabela 5, embora possam ser ditos esperados, a avaliação da perfôrmance dos estados em cada caso, relacionados a essas variáveis, requereria a adoção de um modelo econométrico, ou outra metodologia similar que, no entanto, foge do escopo deste artigo.

\section{Considerações Finais}

A metodologia adotada neste artigo permite, a partir da utilização da abordagem multidimensional da pobreza, o cálculo de um indicador-síntese de pobreza multidimensional, tendo como referência os Objetivos do Desenvolvimento do Milênio. Isto diminui o grau de arbitrariedade quanto à escolha das dimensões relevantes da pobreza multidimensional e, ao mesmo tempo, fornece uma medida de acompanhamento conjunto acerca de se as metas e os Objetivos do Desenvolvimento do Milênio estão sendo alcançados pelos estados brasileiros.

Vale observar que os cálculos foram realizados a partir de uma referência de dados secundários bastante comum e acessível, como aqueles disponíveis no IPEDATA, INEP, DATASUS, entre outras, o que permite, com poucas modificações, um acompanhamento anual, dos Objetivos do Desenvolvimento do Milênio.

De acordo com a metodologia adotada tendo como referência a teoria do conjunto fuzzy, os estados com os maiores índices são aqueles com maior pobreza do ponto de vista multidimensional. Assim, os resultados apontam uma 
delimitação espacial bem definida no país, com os estados das regiões Norte e Nordeste situando-se entre os dez de piores índices, à exceção do estado do Rio Grande do Sul. No outro extremo da distribuição aparecem os estados das regiões Sul e Sudeste, à exceção do estado do Rio Grande do Norte, como aqueles de menores índices de pobreza multidimensional.

Todavia, a despeito do fato de que a descrição dos dados segue um padrão regional, o que pode parecer trivial, a partir das variáveis consideradas para a construção dos indicadores utilizados para avaliar cada meta e, portanto, o Objetivo do Desenvolvimento do Milênio, observou-se que o estado do Rio de Janeiro, por exemplo, aparece como aquele de posição mediana, na distribuição do indicador de pobreza multidimensional entre os estados, o que denota que aquele estado apresenta restrições de funcionamentos muito significativas.

Não obstante, observa-se uma correlação negativa entre o índice multidimensional de pobreza calculado e algumas variáveis que poderiam em princípio ter influência sobre o baixo desempenho no alcance dos Objetivos do Milênio, dos estados das regiões mais pobres do país, Norte e Nordeste, como o PIB, as transferências públicas federais para os estados, o volume dos gastos sociais e os investimentos.

\section{Referências Bibliográficas}

S. Alkire. Dimensions of human development. World Development, 30:181205, 2002.

I. Bagolin e R. P. Ávila. Poverty distribution among the brazilian states: a multidimensional analysis using capabilities and need approaches. In Encontro Nacional de Economia, volume 34, 2006.

R. P. Barros, M. Carvalho, e S. Franco. Pobreza multidimensional no brasil. Technical report, 2006.

L. Canova, M. Grasso, A. Vaglio, E. Di Giulio, S. Migliavacca, S. Lelli, e S. Pareglio. Operationalising senian capability approach by modelling human development. Technical report, Universitá Cattolic del Sacro Cuore, 2005.

B. Cheli e A. Lemmi. A totally fuzzy and relative approach to the measurement od poverty. Economic Notes, 94 (1):115-134, 1995.

G. A. Cohen. Igualdade de que? In M. C. Nussbaum e A. K. en, editores, $L a$ Calidad de Vida. Mexico: FCE, 1996.

F. Comim, I. P. Bagolin, R. Avila, S. Porto Junior, e V. C. Picolotto. Pobreza da insuficiência de renda à privação de capacitações: uma aplicação para a cidade de porto alegre através de um indicador multidimensional. mimeo. 2006.

A. Hagenaars e K. de Vos. The definition and measurement of poverty. The Journal of Human Resources, 23:211-221, 1988.

IPEA. Objetivos do desenvolvimento do milênio: relatório nacional de acompanhamento. Technical report, Instituto de Pesquisa Econômica Aplicada, 2007. 
A. Kageyama e R. Hoffman. Pobreza no brasil: uma perpectiva multidimensional. Economia e Sociedade, 15:79-112, 2006.

G. J. Klir e B. Yuan. Fuzzy sets and fuzzy logic: theory and applications. Prentice Hall PTR, 1995.

H. M. Lopes. Análise de pobreza com indicadores multidimensionais: uma aplicação para o brasil e minas gerais. Master's thesis, CEDEPLAR, 2003.

H. M. Lopes, P. B. R. Macedo, e A. F. Machado. Análise de pobreza com indicadores multidikensionais para o brasil e minas gerais. Technical report, CEDEPLAR, 2004.

M. C. Nussbaum e A. K. Sen. La Calidad de Vida. Fondo de Cultura Económica, 1996.

ONU. Nações unidas declaração do milênio:. Technical report, Organização das Nações Unidas, 2001.

V. Picolloto, F. Comim, I. Bagolin, S. Porto Junior, e R. Ávila. Avaliação multidimensional da pobreza - em exercício piloto para porto alegre e região metropolitana. In Encontro de Economia da Região Sul, 2007.

J. Rawls. A theory of justice. Master's thesis, Havard University, 1971.

I. Robeyns. Justice as fairness and the capability approach. In Cabability Conference, 2004.

I. Robeyns. The cabability approach and welfare policies. In Conference Gender Auditing and Gender Budgeting, 2005.

M. C. Romão. Pobreza: conceito e mensuração. Technical report, Programa Nacional de Pesquisa Econômica, 1993.

P. Salama e B. Destremau. Medidas de pobreza desmedida: economía política de la distribuición del ingreso. LOM Ediciones, 2002.

A. K. Sen. Poverty: an ordinary approach to measurement. Econometrica, 44: 219-231, 1976.

A. K. Sen. Equality of what? In Tanner Lectures on Human Values. Cambridge University Press, 1980.

A. K. Sen. Commodities and capability. Oxford University Press, 1985.

A. K. Sen. Pobreza e fomes: um ensaio sobre direitos e privações. Terramar, 1999.

A. K. Sen. Desenvolvimento como liberdade. Companhia das Letras, 2000.

A. K. Sen e E. Foster. La Desigualdade Económica. Fondo de Cultura Económica, 2001.

J. Verkuilen. Assigning membership in a fuzzy set analysis. Sociological Methods E Research, 33:462-496, 2005. 